\title{
Programas de Ciencias en la educación primaria costarricense: relación entre la gestión de riesgos y el cambio climático
}

\author{
Programs in Costa Rican primary education: \\ Relationship between Risk Management and Climate Change
}

\author{
Jeannette Arauz Muñoz ${ }^{1}$ \\ Cristian Moreira Segura ${ }^{2}$ \\ Claudia Charpentier Esquivel ${ }^{3}$ \\ Tecnológico de Costa Rica, Costa Rica
}

\begin{abstract}
Resumen. La gestión integrada del riesgo de desastres (GIRD), asociada al cambio climático (CC), está estrechamente vinculada con el sector educación. Por su importancia, se analizaron los programas de Ciencias pertenecientes al I y II ciclos del año 2016 del Ministerio de Educación Pública de Costa Rica. El objetivo fue evaluar los elementos curriculares de los contenidos, las metodologías y los recursos didácticos en lo referente a la GIRD asociada al CC (adaptación y mitigación). Dentro de los hallazgos importantes, se resalta que en los programas se reflejan contextos ambientales variados y en diferentes escalas geográficas (local, nacional y global); asimismo, se alude a los conceptos de prevención, riesgos socioambientales, CC, calentamiento global, fenómenos meteorológicos, recursos hídricos y aguas subterráneas, entre otros. La presencia vinculada de estos elementos en los planes denota su actualización con respecto a las principales corrientes teóricas de la GIRD y el CC que se han propuesto y que guían el corpus científico tanto a nivel nacional como mundial. Otros elementos de calidad presentes son: se centra en el estudiante, es coherente en los diferentes años escolares y es flexible. Los resultados de este análisis se utilizarán en una etapa posterior como parte de una investigación más amplia, cuya finalidad es crear una propuesta educativa ambiental para elaborar competencias relacionadas con la GIRD asociada al CC en la escuela Excelencia de Cahuita en Limón, Costa Rica.
\end{abstract}

Palabras clave. Gestión integrada de riesgos de desastres, cambio climático, contenidos programáticos, Ciencias, competencias ambientales.

\begin{abstract}
Integrated management of disaster risk (IDRM), associated with climate change is closely linked to the education sector. Due to its importance, content analysis of the current study programs of Sciences (2016) in I and II cycles of basic general education of the Ministry of Public Education of Costa Rica was carried out for this paper. The objective was to evaluate curricular elements of contents, methodologies and teaching resources in relation to the IDRM associated with climate change (Adaptation and Mitigation). Among the important findings it is highlighted that the programs reflect varied environmental contexts and at different geographical scales (local, national and global); likewise, they allude the concepts of prevention, socio-environmental risks, change climate, global warming, meteorological phenomena, water resources, groundwater, among others. The presence of these elements in the study program implies that they are current and relevant and show they were formulated according to the main theoretical trends of IDRM and climate change, that guide the scientific corpus at national and global level. Other quality elements present are: student-centered, consistent in different school years, and flexible. The results of this analysis will be used later stage as part of a broader investigation in the construction of an environmental education proposal for the creation of competencies related to the IDRM associated with climate change at the Excelencia Cahuita school, Limón, Costa Rica.
\end{abstract}

Keywords. Integrated Disaster Risk Management, climate change, program content, Sciences, environmental competencies.

\footnotetext{
${ }^{1}$ Investigadora independiente. Estudiante del Doctorado en Ciencia Naturales de para el Desarrollo (DOCINADE), Magister Scientae en Geografía, Universidad de Costa Rica. Dirección electrónica: jeannettearauz@gmail.com ; ORCID https://orcid.org/0000-0002-9867-6855

${ }^{2}$ Doctor en Ciencias Naturales para el Desarrollo. Profesor del Doctorado Interuniversitario en Ciencias Naturales para el Desarrollo (DOCINADE), Tecnológico de Costa Rica. Dirección electrónica: cmoreira@tec.ac.cr

${ }^{3}$ Profesora del Doctorado Interuniversitario en Ciencias Naturales para el Desarrollo (DOCINADE), Instituto Tecnológico de Costa Rica. Dirección electrónica: ccharpen@gmail.com
} 


\section{Introducción}

El Grupo Intergubernamental de Expertos sobre Cambio Climático (IPCC por sus siglas en inglés), que elaboró el Informe especial sobre el océano y la criosfera en un clima cambiante (Intergovernmental Panel on Climate Change, 2019), resalta la necesidad de tomar acciones urgentes para hacerle frente al cambio climático (CC) y avanzar hacia el desarrollo sostenible(DS). Una deestasmedidasesintensificarlaeducacióno alfabetización climática, junto con el seguimiento y pronósticos del clima, la búsqueda de fuentes de conocimiento disponibles, el intercambio de datos e información, los mecanismos financieros, la reducción de la vulnerabilidad, la cooperación institucional y la equidad social. Esto conlleva que los estudiantes adquieran conocimientos, destrezas y valores a favor de la vida y el respeto por el medio ambiente. Con lo cual se propiciaría la ejecución de acciones que favorezcan tanto su entorno familiar como el de su comunidad, en especial lo correspondiente a acciones de mitigación y adaptación al CC para prevenir riesgos climáticos.

Dentro del sistema educativo costarricense, el programa de estudios es el referente pedagógico para el quehacer docente, ya que establece la perspectiva epistemológica del conocimiento científico, el enfoque curricular o teórico y la estrategia metodológica para el planeamiento didáctico en el contexto sociocultural y ambiental de la comunidad educativa (Ministerio de Educación Pública [MEP], 2017). Los programas vigentes del Ministerio de Educación Pública de Costa Rica en la asignatura de Ciencias para I y II ciclos de la educación general básica (EGB) fueron aprobados mediante el Acuerdo n. ${ }^{-}$03-30-2016 (MEP y CSE, 2016). Estos se reestructuraron bajo los lineamientos de la Organización de las Naciones Unidas para la Educación, la Ciencia y la Cultura (Unesco, por sus siglas en inglés), según las pautas de la
Educación para el Desarrollo Sostenible (EDS) y de acuerdo con la visión científica "Educar para una nueva ciudadanía" (MEP, 2015a), lo cual representa un cambio curricular cualitativo para el país, porque significa que muchos temas propios de la sostenibilidad están presentes en los planes de estudio. De acuerdo con la postura del Ministerio, la educación científica que se impulsa está fundamentada en tres pilares filosóficos: el humanismo, el constructivismo y el racionalismo, los cuales a su vez son las bases epistemológicas de la política educativa del país (MEP, 2016).

No obstante, aunque la "Educación ambiental fue formalmente incorporada como un componente del currículum escolar en el 2000" (Unesco, 2015, p. 44), el abordaje en el aula de los conceptos interrelacionados con la gestión del riesgo de desastres (GRD) y el CC para el desarrollo de competencias es poco frecuente. La mayoría de las veces se quedan en el papel como contenidos y criterios de evaluación prescriptivos, que guían al docente en su práctica profesional, sin impulsar un cambio que reduzca la vulnerabilidad por los riesgos climáticos y aumente la capacidad adaptativa y la resiliencia de los niños.

En el Informe hacia una Centroamérica más resiliente, Girot y Perea afirman que "una armonización delas agendas de trabajo en materia de gestión de riesgos de desastres y adaptación al cambio climático podría beneficiarse de un esfuerzo de fortalecimiento de las plataformas regionales y nacionales de provisión de servicios de información climática para una participación ciudadana informada y oportuna" (2019, p. 24). Desde la posición del autor de este artículo, esta "armonización" no escapa del ámbito educativo. A partir de esta realidad, se requiere que la GRD y elCC, como nociones científicas fundamentales y complejas de los problemas mundiales críticos del presente, no solo estén incluidos como 
contenidos temáticos, sino que se interrelacionen y se desarrollen bajo un enfoque de educación sociocrítico y científico. Con este, se busca formar competencias ambientales orientadas a la acción significativa y transformativa en los niños, más allá de las aulas. Dentro del marco anterior, el objetivo general de esta investigación es evaluar los contenidos curriculares, las metodologías y los recursos didácticos presentes en los programas de Ciencias de la educación primaria costarricense (I y II ciclos), sobre la GRD y el CC (mitigación y adaptación). Se parte de que una revisión y análisis de los planes de estudio vigentes son determinantes para comparar el estado o función actual de los programas con el estado o función potencial o ideal de estos.

\section{Antecedentes}

De acuerdo con el III Informe País 2015 de Costa Rica (Ministerio de Planificación Nacional y Política Económica [Mideplan], 2015), después de realizar un balance sobre el cumplimiento de los Objetivos de Desarrollo del Milenio, se evidenció que todavía persistían desafíos para alcanzar el desarrollo de su población. Se señalaba que, en materia de educación y ambiente, "El método que se utiliza para alfabetizar, está desactualizado y no prepara para generar competencias entre la población, para que se mantengan dentro del sistema educativo"; además, se destacaba la "Falta de implementación y regulación de políticas como Ordenamiento Territorial, Ley de Gestión de Residuos Sólidos y política de CC" (Mideplan, 2015, p. 145). En consecuencia, en las directrices del Plan Nacional de Desarrollo 20152018 Alberto Cañas Escalante (2015-2018), se estableció el componente de Educación ambiental y se planteó la inquietud de reformular los programas de estudio para mejorar las temáticas vinculadas "con el currículum, metodologías de enseñanza que promuevan conocimientos y el desarrollo de habilidades y destrezas con el propósito de formar personas productivas que ejerzan la ciudadanía y participen activamente en el desarrollo del país" (Mideplan, 2014, p. 217).

Los lineamientos del Viceministerio Académico, contemplados en el documento "Educar para una nueva ciudadanía" (MEP, 2015a), establecieron -bajo una nueva propuesta curricular - los perfiles de salida específicos del estudiante de I, II y III ciclos de la EGB para el desarrollo de habilidades basadas en tres tipos de aprendizaje: cognitivo, socioafectivo y actitudinal en el proceso de alfabetización científica. Con esto, se resalta el papel preponderante y la responsabilidad de la escuela, así como el empoderamiento de la comunidad educativa para generar una educación contextualizada (MEP, 2015a).

El MEP define habilidad como la capacidad del estudiante para realizar distintas tareas y enfrentar situaciones adversas de la vida cotidiana. Educar en habilidades fomenta la formación continua e integral de la persona a partir de la apropiación del conocimiento sistematizado, lo cual lleva a proponer un aprendizaje propio con pensamiento crítico, creativo y de valores (Alfaro-Rodríguez, 2015). La propuesta educativa oficial se fundamenta en tres elementos teóricos o competencias clave, de acuerdo con la Organización para la Cooperación y el Desarrollo Económicos (OECD, por sus siglas en inglés, 2017): la EDS, la ciudadanía planetaria con identidad nacional y la ciudadanía virtual con equidad social, las cuales son transversales en todos los temas que integran el currículum (MEP, 2015b).

Según concluye la OECD, en el informe especial de educación, la reforma curricular costarricense es un gran logro porque está enfocada en la formación de competencias, lo cual es una manera positiva de promover el aprendizaje y el desarrollo de procesos mentales de tipo superior (OCDE, 2017). Sin embargo, señala situaciones que se deben solucionar para tener éxito en el 
cambio curricular: la capacitación en enseñanza y evaluación de competencias de los docentes, así como laborar durante todo el día en la escuela. La nueva visión curricular se basó en la propuesta hecha por la Fundación Omar Dengo de Costa Rica (FOD) sobre las Competencias del Siglo XXI, mediante el Proyecto ATC21s de carácter internacional (Fundación Omar Dengo de Costa Rica, 2014; Marope, Mmantsetsa, Griffin, Patrick, Gallagher y Carmel, 2018). La aplicación educativa de los planes de estudio es reciente, tiene solo 4 años; por ello, el MEP espera que se evalúe su implementación pasados cinco años (MEP y CSE, 2016).

Las directrices educativas del 2016 comprendían cuatro dimensiones y trece habilidades específicas; estas últimas se subdividen, a su vez, en veinte indicadores para el perfil de salida de cada ciclo escolar. Las dimensiones son: 1. Maneras de pensar (pensamiento sistémico, crítico, aprender a aprender, resolución de problemas, creatividad e innovación transformadora del entorno para modificar el modelo actual de desarrollo); 2. Nuevas formas de vivir en el mundo (ciudadanía global y local, estilos de vida saludable, responsabilidad personal y social, toma de decisiones y resolución de problemas socioambientales bajo un proceso de innovación, vida y carrera); 3. Formas de relacionarse con otros (colaboración y comunicación); y 4. Herramientas para integrarse al mundo (apropiación de tecnologías digitales y manejo de la información) (MEP, 2020, pp. 2-3).

Bajo este nuevo enfoque educativo, la visión del DS está presente en los programas de estudio y sigue los lineamientos de la Unesco (Unesco, 2010, 2014), mediante la definición de cuatro categorías: los contenidos para el aprendizaje; las estrategias en la mediación pedagógica y ambientes pedagógicos adecuados; los resultados del aprendizaje, y la transformación social mediante el desarrollo de habilidades (conocimientos, destrezas y valores) que conlleven a una nueva relación con el entorno mediante el empoderamiento del estudiante (MEP, 2015b). El desarrollo de estas categorías se hace a través del tema: Cultura ambiental para el desarrollo sostenible, que busca llevar al alumno hacia conductas apegadas al quehacer científico y con una perspectiva ética mediante las cuatro categorías, habilidades e indicadores específicos mencionados.

Por lo tanto, el desarrollo educativo de las habilidades, desde la perspectiva del MEP, se hace porque se conceptualiza la educación ambiental como el instrumento adecuado para fomentar y construir una cultura en la población. El fin es lograr el DS mediante la comprensión del entorno y sus diferentes sistemas (político, social, cultural y ambiental). Se parte del entendimiento, actitudes, acciones y el respeto de los estudiantes hacia el entorno social y ambiental; esto se desarrolla con el aporte de soluciones a los problemas que les afecten (MEP, 2016). Recientemente, el MEP promulgó - bajo la circular DM-0004-01-2020 - los Lineamientos generales para el inicio del curso lectivo 2020 "Año 2020: transformación curricular, una apuesta por la calidad educativa" (Acuerdo CSE-03-012020). Allí se oficializaron las nuevas directrices para la elaboración del planeamiento didáctico ("habilidad y su conceptualización, pautas para el desarrollo de los aprendizajes esperados, indicadores de aprendizaje y niveles de logro de las habilidades del estudiante") (MEP, 2020).

\section{Metodología}

Se analizó uno de los programas vigentes del I Ciclo (1.o, 2.o y 3.er año) y II Ciclo (4.o, 5.o y 6.o año) deCiencias, empleados por los docentes para la enseñanza de la temática de GRD, relacionadas con el CC. Metodológicamente, para la revisión y análisis curricular, se emplearon las técnicas de 
análisis de contenido (Hernández, Fernández y Baptista, 2014) y el método de análisis sugerido por la Unesco para encontrar la sostenibilidad en los planes de estudio existentes (Unesco, 2014). Junto con lo anterior, se consultaron revistas científicas, libros, circulares y documentos del MEP e información en la web relacionados con el tema. El análisis demandó la identificación de conceptos básicos en los planes de estudio (sobre ciencia climática, la GRD y el recurso hídrico), con base en el modelo de sostenibilidad de la Unesco y en el marco de trabajo para la alfabetización ambiental (Hollweg, Taylor, Bybee, Marcinkowski, McBeth y Zoido, 2011).

Para la revisión y análisis documental, se establecieron categorías que permitieron organizar e interpretar conceptualmente la información y facilitaron su presentación. Este se revisó a partir de los aspectos que atañen a la EDS y que establece la Unesco. Sin embargo, en los programas de estudio emplean una nomenclatura diferente (Chen-Quesada et al., 2018), a saber: 1. Contenidos de aprendizaje (ejes temáticos); 2. Resultados del aprendizaje (criterios deevaluación);3.Pedagogía y el entorno de aprendizaje (situaciones de aprendizaje), y 4. Transformación social (habilidades de los alumnos); elementos que se usaron para elaborar la propuesta curricular vigente (MEP, 2015b), siguiendo la hoja de ruta para la ejecución del Programa Acción Mundial de EDS (Unesco, 2014), ver tabla 1.

Para lo anterior, se empleó un sistema de codificación con el fin de marcar las palabras y secciones clave del plan de estudio en formato PDF. Se utilizó la herramienta de búsqueda del programa informático Nitro Pro 9, posteriormente, con el comando "marcar texto", se resaltaron en amarillo las palabras encontradas y se subrayaron en verde aquellas secciones de interés temático. A continuación, se hicieron comentarios al margen de los programas como parte del análisis de contenido. Se generaron varias matrices para el ordenamiento, clasificación, categorización e interpretación de los datos encontrados, que se

\section{Tabla 1}

Categorías de clasificación conceptual para identificar, organizar, interpretar, analizar y presentar la información de los planes de estudio de Ciencias de I y II ciclos

Categoría $\quad$ Eje temático $\begin{aligned} & \text { Criterios de } \\ & \text { evaluación }\end{aligned} \begin{aligned} & \text { Situaciones de } \\ & \text { aprendizaje }\end{aligned}$

1. Relacionado con la GRD.

2. Relacionado con el CC y la GRD.

3. Relacionado con la interacción de los conceptos de GRD, CC y variabilidad climática (VC).

4. Relacionado con el clima.

5. Relacionado con el CC.

6. Relacionado con el recurso hídrico (RH).

$\begin{array}{lll}X & X & X \\ X & X & X \\ X & X & X\end{array}$

$\begin{array}{lll}X & X & X\end{array}$

$\begin{array}{lll}X & X & X\end{array}$

$x \quad x \quad x$

Nota. Arauz, Charpentier y Moreira, 2020. 
cruzaron y validaron con diferentes documentos relativos al tema de estudio. El producto final fue el levantamiento y análisis de los contenidos curriculares o programáticos, metodologías y recursos didácticos sobre la enseñanza relacionada con la temática mencionada para la asignatura de Ciencias en los años escolares de primero a sexto, descritos en los programas de estudio.

\section{Resultados y discusión}

Comparación de las definiciones de cambio climático, variabilidad climática y gestión integrada del riesgo de desastres, incluida en los planes de estudios con las definiciones establecidas por el IPCC

Después de un análisis comparativo de los términos cambio climático y gestión del riesgo de desastres, con las conceptualizaciones dadas por los expertos del IPCC (IPCC, 2013), se encontró lo siguiente:

En los planes de estudio, la palabra cambio climático está descrita parcialmente y sigue la definición dada por la Convención Marco de las Naciones Unidas sobre el Cambio Climático (CMNUCC). En la definición oficial del MEP, dicho vocablo se refiere a los cambios en el clima causados por la intervención directa o indirecta de las acciones humanas, que perturban la composición natural de la atmósfera mundial y aumentan la concentración de gases de efecto invernadero (MEP, 2016). Se omitió la segunda parte de la explicación dada por la CMNUCC: hay que sumar las variaciones climáticas naturales. Por su parte, el IPCC (2013) precisa el término clima como el registro estadístico de la variabilidad natural del comportamiento de sus elementos (precipitación pluvial, temperatura, humedad atmosférica, velocidad del viento) durante períodos comparables (al menos 30 años) y cuyas causas son forzamientos externos (variaciones solares, erupciones volcánicas o cambios antropógenos). En resumen, "la
CMNUCC diferencia, pues, entre el cambio climático atribuible a las actividades humanas que alteran la composición atmosférica y la variabilidad climática atribuible a causas naturales" (IPCC, 2013, p. 188).

Con este análisis, se colige que, en los planes de estudio de Ciencias de I y II ciclos, se presenta una propensión pedagógica hacia la acepción de cambio climático causado por el ser humano, lo cual permite enfocarse en los procesos de adaptación transformativa y resiliencia, no solo debido a las variacionesnaturales y quizás, por eso, se "omite" en la definición. Esta presencialidad de los conceptos es un rasgo de calidad en los planes de Ciencias de I y II ciclos que está acorde con las políticas nacionales e internacionales, las cuales enfatizan que la educación es el mejor instrumento de sensibilización para producir un cambio esencial en el comportamiento humano hacia un mundo sostenible. Al respecto, Stabbak (2016) menciona que el currículum, como documento técnico, refleja "también un acuerdo político y social más amplio acerca de lo que la mayoría de la sociedad considera más valioso lo que es suficientemente importante como para transmitirlo a los niños" (p. 8).

Así, al comparar el vocablo GRD presente en los planes de estudio y el propuesto por el IPCC, se concluye que son convergentes en su definición. En el documento curricular oficial, se utiliza el término delimitado en la Ley Nacional de Emergencias y Prevención de Riesgos n. ${ }^{\circ}$ 8488, que lo define como un proceso para la reducción de las condiciones de vulnerabilidad de los sistemas naturales y socioeconómicos del país, incluyendo las actividades productivas, líneas vitales, servicios y el ambiente (ALRCR, 2006). Además, en el glosario del IPCC, aparte del vocablo gestión de riesgos, se describe el concepto gestión de riesgos de desastre, el cual refiere a procesos para mejorar la comprensión del riesgo; lo cual es totalmente coincidente 
con el término que aparece en el glosario de los planes de estudio.

Estructuración curricular y operacionalización del plan de estudios de Ciencias de I y II ciclos

Desde el punto de vista estructural, los programas están en un documento compuesto por nueve apartados. Consta de una introducción en la cual se aboga por una "Educación científica para una nueva ciudadanía en el contexto del DS". La segunda parte describe la fundamentación filosófica y epistemológica de la naturaleza del conocimiento científico que se compone de tres apartados; además, presenta la visión curricular y la estrategia metodológica fundada en la indagación. La tercera sección es el perfil de salida del estudiantado y del docente; la cuarta refiere a la nueva propuesta del "diseño curricular" con la descripción detallada de los programas de estudio de primero a sexto año. Finalmente, las secciones quinta, sexta, séptima, octava y novena corresponden a las referencias, los créditos, un anexo titulado "El planteamiento de preguntas", un artículo sobre la transversalidad en los programas de estudio y un glosario, respectivamente (MEP, 2016, pp. 2-3) (Fig. 1). La investigación se centró en el análisis de las secciones tercera y cuarta del plan de estudios de II Ciclo, aunque se abordó globalmente del documento.

Desde la óptica del diseño curricular, los planes de estudio comprenden cuatro elementos: nivel, que es el año escolar o ciclo instructivo obligatorio, en este caso corresponde al I y II ciclos de la EGB; ejes temáticos (o contenidos de aprendizaje), temas de la unidad de carácter integrador mediante los que se organizan y articulan los saberes inherentes de Ciencias (MEP, 2016); criterios de evaluación (o resultados de aprendizaje), estos permiten establecer la clase y grado del aprendizaje que se espera del estudiante en relación con las habilidades $\mathrm{y}$ actitudes presentes "en los objetivos, contenidos curriculares, habilidades específicas o competencias" (Torres-Arias et al., 2015, p. 4); y las situaciones de aprendizaje (Pedagogía y entornos de aprendizaje), que son las diferentes actividades descritas secuencialmente, por medio de las cuales se evalúa el proceso educativo del alumno con respecto a la elaboración y apropiación del conocimiento de los sistemas físicos, biológicos, psicológicos y sociales, para lo que se emplea el enfoque metodológico basado en la indagación (MEP, 2016).

La operacionalización del plan en el aula sehace a partir del trabajo cotidiano estudiante-docente con los elementos curriculares-estructurales mencionados. El trabajo que el docente realiza mediante el uso de la pregunta-problema en la mediación pedagógica es fundamental para el desarrollo de la competencia, y se debe abordar empleando la estrategia metodológica basada en la indagación o el "aprendizaje por investigación, Inquiry-Based Learning (IBL) o Inquiry-Based Science Education (IBSE)" (MEP, 2017; Romero-Ariza, 2017, p. 288).

En los planes, se identificaron tres ejes temáticos: "I. Los seres vivos en entornos saludables, como resultado de la interacción de aspectos biológicos, socioculturales y ambientales; II. Uso sostenible de la energía y los materiales para la preservación y protección de los recursos del planeta; y III. Interrelaciones entre las actividades que realiza el ser humano a nivel local y global, con la integridad del planeta Tierra y su vinculación con el Universo" (MEP, 2016, pp. 38-39). A juicio de las autoridades del MEP, esta propuesta curricular es flexible, ya que puede ser modificada por el docente según las características de la comunidad educativa en donde se desarrolle su práctica y valorando el horario o el tipo de centro escolar, así como la disponibilidad de recursos económicos, sociales o naturales. Con esto, se generaría un proceso de aprendizaje inclusivo para el estudiante (MEP, 2016). 


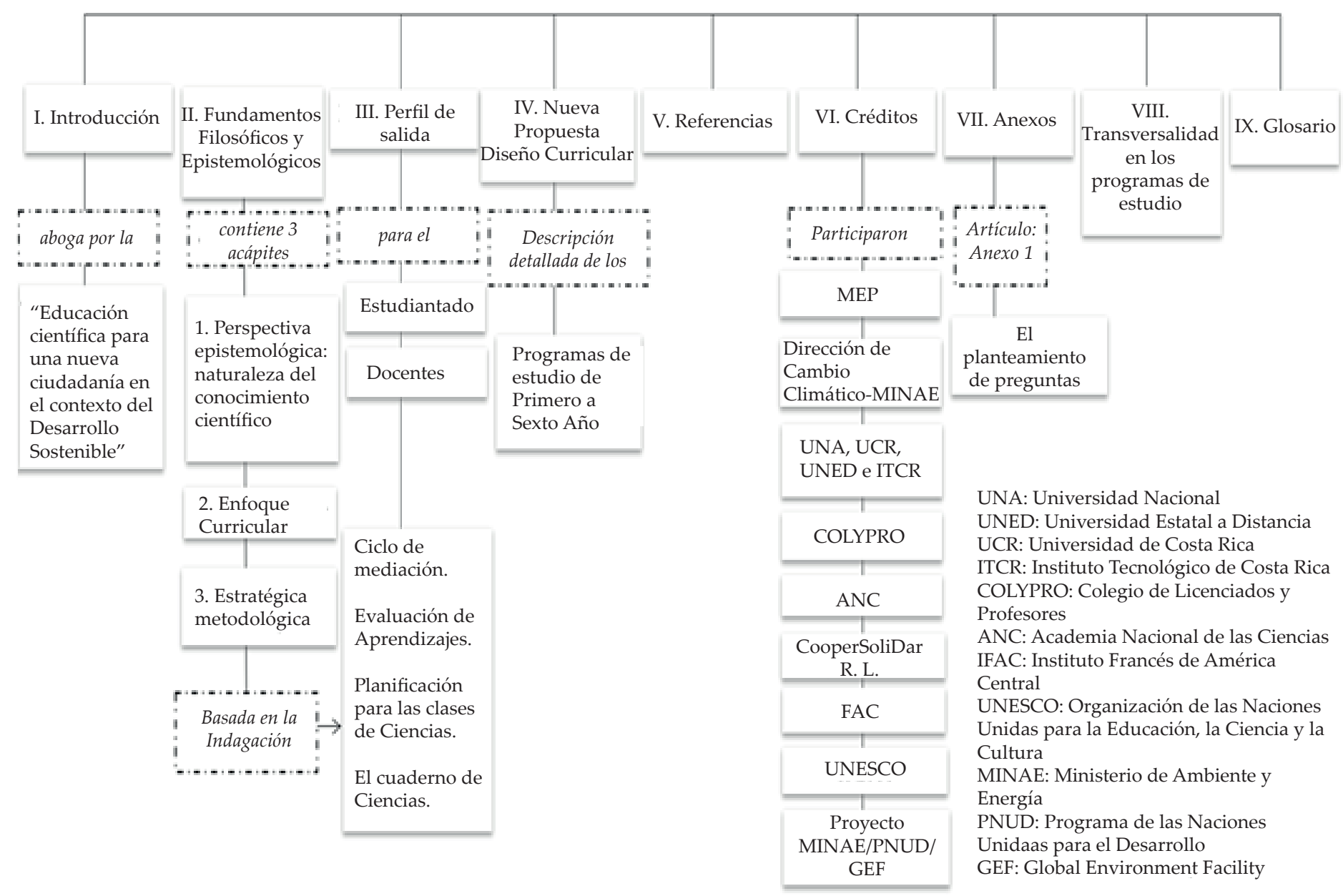

Figura 1. Estructura del documento oficial de los programas de estudio de Ciencias de I y II ciclos del MEP. Elaborado por Arauz, Charpentier y Moreira, 2020. 
Losprogramasdeestudioparacada año, es decir de primero a sexto, cuentan de manera uniforme con tres ejes temáticos, que se subdividen en 33 secciones por cada ciclo. El total de 66 secciones, denominadas criterios de evaluación son producto de la subdivisión de cada uno de los tres ejes temáticos en el que se fracciona el plan: para el I y II ciclos, el primer eje, hay 32 (48.48\%); 22 (33.33\%) para el segundo y $12(18.18 \%)$ para el tercero. Se evidencia que el primer eje temático tiene el mayor peso por la cantidad de secciones; en cambio, el tercero, apenas 12, esto supone que el docente requiere de un nivel de profundidad y tiempo de desarrollo mayor con respecto a los demás ejes para su abordaje práctico. Un análisis más detallado de la estructuración del plan muestra la cantidad de secciones por eje temático por ciclo y año escolar: el primer eje temático presenta más apartados, con un total de 17 y 15 para el II Ciclo; el segundo eje tiene 10 en el I Ciclo y 12 en II Ciclo; finalmente, para el III Ciclo, cuenta con 6 secciones para I y II ciclos (ver tabla 2).
El elemento curricular eje temático es la columna vertebral del programa desde la EDS; se considera el contenido de aprendizaje integrador que guía el quehacer docente para cada periodo durante el ciclo lectivo, mediante la organización y la concatenación de los saberes intrínsecos de la disciplina. Este componente concuerda con el grado de profundización que se requiere en los diferentes niveles educativos y se apega al desarrollo biológico, cognitivo y psicoemocional del estudiante, los cuales a su vez definen los aprendizajes esperados (MEP, 2016). Las temáticas están interrelacionadas y cambian por año escolar y periodo para ser estudiadas durante el ciclo lectivo de 36 semanas. Los docentes deben abordar las situaciones de aprendizaje en un lapso de dos a tres semanas (MEP, 2016).

La tabla 3 resume la cantidad de criterios de evaluación por eje temático y año escolar del plan de estudios para ambos ciclos. Se visualiza que el primer eje temático presenta mayor cantidad de criterios de evaluación (96) con un $48.48 \%$ y el tercer eje, la menor cantidad, con tan solo 36,

Tabla 2

Cantidad de secciones del plan de estudios de Ciencias de I y II ciclos de 2016 por año escolar y eje temático

\begin{tabular}{|c|c|c|c|c|c|c|c|c|}
\hline \multirow{4}{*}{ Eje temático } & \multicolumn{6}{|c|}{ Año escolar } & \multirow{4}{*}{ Total } & \multirow{3}{*}{$\%$} \\
\hline & \multicolumn{3}{|c|}{ I Ciclo } & \multicolumn{3}{|c|}{ II Ciclo } & & \\
\hline & \multicolumn{6}{|c|}{ Cantidad de secciones } & & \\
\hline & 1.0 & 2.o & 3.0 & 4.0 & 5.0 & 6.0 & & \\
\hline I & 6 & 6 & 5 & 5 & 5 & 5 & 32 & 48.48 \\
\hline II & 3 & 3 & 4 & 4 & 4 & 4 & 22 & 33.33 \\
\hline III & 2 & 2 & 2 & 2 & 2 & 2 & 12 & 18.88 \\
\hline Subtotal & 11 & 11 & 11 & 11 & 11 & 11 & 66 & \\
\hline Total & & 33 & & & 33 & & 66 & 100 \\
\hline
\end{tabular}

Nota. Arauz, Charpentier y Moreira, 2020. 
o sea, un $18.18 \%$. Además, ambos ciclos tienen 33 criterios de evaluación en cada nivel.

Programas para el I Ciclo (primero, segundo y tercer año)

Con el análisis de los programas de estudio, se identificó que el desarrollo de habilidades asociadas al modelo de DS, de acuerdo con el diseño curricular del MEP, se establece de manera holística, articulada y progresiva en los tres diferentes ejes temáticos mencionados entre los ciclos escolares I y II de la educación general básica, bajo la formación de destrezas vinculadas con el quehacer científico. De la revisión y análisis documental, se obtiene que el perfil de salida específico para el I Ciclo, relativo a los saberes de la asignatura de Ciencias, es a partir de la dimensión denominada Nuevas formas de vivir en el mundo, y bajo las habilidades Estilos de vida saludable y responsabilidad personal y social, que se mencionan las temáticas sobre "situaciones de emergencia", "cuidado del entorno sociocultural y natural" y "riesgos socioambientales". De esta manera, se presentan los indicadores. Estos, según la terminología oficial, son criterios de evaluación o resultados de aprendizaje que dan las pautas para el desarrollo de la habilidad en el perfil de salida del estudiante. En un análisis comparativo, se concluye que estos criterios de evaluación coinciden con dos competencias generales en el dominio de la alfabetización ambiental contempladas en el modelo teórico de la Asociación Norteamericana de Educación Ambiental (NAAEE, por sus siglas en inglés) (Hollweg et al., 2011): la identificación y el análisis de problemas ambientales, como parte de un proceso educativo que permite a una persona una adecuada cultura sostenible.

Por lo tanto, primero, el estudiante debe hacer un reconocimiento de los diferentes tipos de riesgos que impactan a todos los seres vivos. Con ello, se espera que, según la taxonomía del sistema de cognición propuesta por Marzano y Kendall (2007), integre $\mathrm{y}$ entienda estos conceptos $\mathrm{e}$ interprete dicha información a partir de su conocimiento previo. Después, con un nivel analítico más profundo y riguroso, diferencie

Tabla 3

Cantidad de criterios de evaluación del plan de estudios de Ciencias 2016 para I y II ciclos por año escolar y eje temático

\begin{tabular}{|c|c|c|c|c|c|c|c|c|}
\hline \multirow{4}{*}{ Eje temático } & \multicolumn{6}{|c|}{ Año escolar } & \multirow{3}{*}{ Total } & \multirow{3}{*}{$\%$} \\
\hline & \multicolumn{3}{|c|}{ I Ciclo } & \multicolumn{3}{|c|}{ II Ciclo } & & \\
\hline & \multicolumn{6}{|c|}{ Cantidad de secciones } & & \\
\hline & 1.0 & 2.0 & 3.0 & 4.0 & 5.0 & 6.0 & & \\
\hline I & 18 & 18 & 15 & 15 & 15 & 15 & 96 & 48. 48 \\
\hline II & 9 & 9 & 12 & 12 & 12 & 12 & 66 & 33.36 \\
\hline III & 6 & 6 & 6 & 6 & 6 & 6 & 36 & 18.18 \\
\hline Subtotal & 3 & 11 & 11 & 11 & 11 & 11 & 66 & \\
\hline Total & 33 & 33 & 33 & 33 & 33 & 33 & 198 & 100 \\
\hline
\end{tabular}

Nota. Arauz, Charpentier y Moreira, 2020. 
entre las opiniones, actitudes y acciones que estas tienen sobre la salud individual y comunal, para trasladar dicho conocimiento a nuevos contextos, de forma que lo incorpore de manera significativa a su aprendizaje de memoria a largo plazo y para la vida. Este objetivo va aunado a la habilidad "responsabilidad personal y social" que se presenta en estos niveles escolares, con la cual se propone que el alumno comprenda las distintas medidas de protección existentes para enfrentar situaciones de emergencia originadas por eventos naturales o provocadas por la acción humana (MEP, 2016, p. 32).

Por su parte, el perfil de salida plantea que el alumno participe en acciones para mejorar y proteger el entorno natural y sociocultural de su escuela y de la comunidad donde vive; además, que describa las actividades que ayuden a solucionar las diferentes problemáticas ecológicas de su localidad (MEP, 2016). El MEP realizó una adecuación curricular para el nivel preescolar, pues considera clave que la niñez desarrolle actitudes de respeto y cuidado del medio ambiente local y global, las cuales favorezcan desde la primera infancia los procesos de DS. Está comprobado que, entre las edades de 0 a 4 años, los niños se hacen muy sensibles en relación con su entorno, gracias a su natural curiosidad (Espinoza-Villalobos, 2018).

\section{Programas para el II Ciclo (cuarto, quinto y sexto año)}

La revisión documentaly el análisis de contenido del programa de Ciencias para el II Ciclo, según el perfil específico de salida del estudiante, parte de la dimensión denominada Nuevas formas de vivir en el mundo, la cual está asociada a las habilidades de Responsabilidad personal y social y estilos de vida saludable, respectivamente, mencionadas desde el segundo año escolar en las temáticas de estudio, bajo el uso de indicadores. En este caso, el planteamiento busca promover en el estudiante "medidas de prevención y protección ante situaciones de riesgo presentes en la comunidad" (MEP, 2016, p. 31). Es decir, que pueda utilizar el conocimiento o la información aprendida, así como métodos, conceptos y teorías en situaciones de la vida real, para resolver tareas y problemas mediante destrezas y saberes; además, se proyecta su participación en acciones para resolver problemas ambientales propios de su entorno comunitario (MEP, 2016).

Tal y como afirma Ireland (2013, p. 32), se debe asegurar que "los indicadores cubran los dominios cognitivos (pensamiento o conocimiento y comprensión), afectivo (sentimientos $\mathrm{o}$ actitudes $\mathrm{y}$ valores) $\mathrm{y}$ psicomotor (hacer o habilidades y procesos)". Al hacer un análisis comparativo de los verbos usados "promueve" y "comprende", bajo el desarrollo de la dimensión Nuevas formas de vivir en el mundo, redactados en tercera persona del singular, de acuerdo con las directrices actuales del MEP (2017), se concluye que estos criterios de evaluación corresponden específicamente con dos competencias generales en el dominio de la alfabetización ambiental del modelo teórico de la NAAEE (Hollweg et al., 2011). Estas influyen para que una persona desarrolle un comportamiento ambientalmente responsable. Además, los dos verbos mencionados se vinculan con la cuarta competencia general de la NAAEE, que se refiere a la capacidad del estudiante para proponer y justificar acciones de cara a los problemas ambientales, la cual se asocia con el nivel 4 denominado Aplicación del conocimiento significativamente, del Sistema de Clasificación Cognoscitivo de Marzano y Kendall (2007). En este caso, el estudiante debe usar el conocimiento de manera significativa, pero tiene que trascender y llevarlo al plano de la acción competente (Ireland, 2013) mediante la destreza en su ámbito de acción o contexto. El mismo autor señala que "La acción competente se centra en el pensamiento crítico e integrador en relación con las decisiones contextuales 
tomadas como parte de la participación ciudadana; el desarrollo de la competencia personal y la capacidad, y de la competencia colectiva" (p. 99).

Por su parte, en el perfil de salida del estudiante, bajo la dimensión Estilos de vida saludable, el empleo del verbo "comprende" se vincula con el nivel 2 de comprensión (Marzano y Kendall, 2007), porque involucra la adquisición e integración del saber. Así, se establece que puede entender los diferentes patrones de consumo desmedidos que repercuten negativamente en la salud individual y comunal (MEP, 2016) y se relaciona con la competencia general del modelo de la NAAEE, la cual define a la persona bioalfabetizada como aquella capaz de identificar los problemas ambientales.

Por lo tanto, el proceso educativo planteado en el plan de estudios muestra que, desde el I Ciclo, el estudiante debe reconocer los diferentes tipos de riesgos que impactan a todos los seres vivos, y trasladar el conocimiento a nuevos contextos e incorporarlos de manera significativa a su aprendizaje de memoria de largo plazo y para la vida, según lo propuesto por la taxonomía del sistema de cognición de Marzano y Kendall (2007). Con ello, se espera que el alumno integre y entienda estos conceptos, interprete dicha información a partir de su conocimiento previo, experiencias y representaciones sociales, para después, en el II Ciclo, con un nivel analítico más profundo por su desarrollo biológico y psicoemocional, participe en actividades en las cuales presente soluciones para resolver problemas socioecológicos en su contexto geográfico inmediato. Lo anterior será posible cuando, aparte de su conocimiento sobre la temática, tenga una fuerte convicción sobre su importancia y su gestión para alcanzar tanto su bienestar como el de las demás personas de su comunidad, del país y del mundo entero.
En las tablas 4, 5 y 6, se resumen las diferentes categorías de análisis y conceptos encontrados en el programa de estudio de Ciencias para el II Ciclo por año escolar y por eje temático. Los resultados muestran que, en el programa de cuarto año, de los tres ejes temáticos, en 20 de sus 33 criterios de evaluación se encuentran presentes los conceptos y categorías de análisis bajo estudio, lo que corresponde a un 60.60 $\%$. No obstante, en 13 criterios (39.39\%) no se mencionan. Si el análisis se hace por eje temático, los hallazgos son: el I eje tiene $15 \%$ de presencia de los conceptos y categorías de análisis; el II eje, $55 \%$, y el III eje presenta un $30 \%$. Se resalta que en el criterio N.1 III-1.2, en la sección de situaciones de aprendizaje, se establece una relación entre todos los temas bajo análisis, es decir, GRD, CC y RH.

Respecto al quinto año, en 18 de 33 criterios de evaluación de los tres ejes temáticos, se encuentran presentes los conceptos y categorías de análisis bajo estudio, lo cual representa un $54.54 \%$, mientras que en 15 criterios no se mencionan (45.46\%). Si el análisis se hace sobre la presencia de los conceptos y categorías de análisis por eje temático, los resultados son: el primer eje tiene 6 criterios, lo que representa un $18.18 \%$; el segundo eje temático cuenta con 9 criterios, con un $27.27 \%$, y el tercero tiene un $9.09 \%$ con 3 criterios de evaluación. Hay 15 criterios en los que no aparecen los conceptos y criterios bajo estudio ( $45.46 \%$ ).

Finalmente, en el programa de sexto año, los conceptos y categorías de análisis bajo estudio se encuentran presentes en 18 de los 33 criterios de evaluación de los tres ejes temáticos. Esto representa un $54.54 \%$ y hay 15 criterios de evaluación en los cuales no se incluyen, es decir, un $45.45 \%$. Si el análisis se hace por presencia de los conceptos y categorías de análisis por eje temático, los hallazgos son que el primer eje menciona 6 criterios, lo que representa un 18.18 
$\%$; el segundo posee 9 criterios que corresponden a un $27.27 \%$, y el tercero presenta 3 criterios, esto es un $9.09 \%$, los cuales suman un total de $54.54 \%$. Además, hay 15 criterios de evaluación repartidos en 9,3 y 3 para el primero, segundo y tercer ejes, respectivamente, en los que no se encuentran los conceptos y criterios bajo estudio (45.45\%) (ver tablas 4, 5 y 6).

Sobre los recursos didácticos en los Programas de Estudios de Ciencias de I y II ciclos

De acuerdo con la nueva propuesta de reforma curricular, los docentes deben centrarse en el proceso de mediación pedagógica siguiendo el enfoque educativo basado en la indagación y el instrumento de planificación de más utilidad que facilita la práctica didáctica se llama "secuencia didáctica". Este consiste en organizar conceptual y metodológicamente un tema de estudio, que oriente todas las acciones educativas necesarias para resolver un problema científico y parte de la indagación (MEP, 2009). Para desarrollar las secuencias didácticas, el educador debe considerar los entornos naturales y humanos, los recursos disponibles en el ambiente, los materiales de laboratorio y el uso de la tecnología (MEP, 2016). Con respecto a los materiales, el MEP refiere al educador al uso de la Caja de Herramientas en el sitio web oficial de la institución. Allí se encuentran algunos recursos didácticos en diferentes formatos (videos, documentos y enlaces) que son los insumos para el desarrollo de su lección; sin embargo, se señala que actualmente se están clasificando (MEP, 2020). Es responsabilidad de los docentes seleccionar los recursos didácticos bibliográficos (incluyen libros de texto $\mathrm{e}$ internet) o virtuales, pero el MEP recomienda que deberán ser principalmente materiales de desecho y reciclaje que provengan de los hogares de los estudiantes para evitar que se incurra en gastos extra (MEP, 2009).
Sumado a lo anterior, la Unesco (2012) identifica, dentro de los 10 aspectos fundamentales para una educación de calidad, que los docentes empleen una amplia gama de procesos pedagógicos y de aprendizaje. Durante la fase de planificación, deben considerar, en la selección de recursos didácticos, aquellos que les ayuden tanto a superar los desafíos en el salón de clases o fuera de este, como a mantener la atención e interés del alumno por profundizar el conocimiento acerca del objeto de estudio y estimular la creatividad (MEP, 2016). Algunos de los recursos didácticos, materiales y técnicas identificados en los programas de estudio para el desarrollo de la mediación pedagógica son los siguientes: cuaderno de Ciencias, recibos de electricidad y agua, presentación de especialistas locales (CNE, Municipalidad, etc.) y de la familia, redacción de proyectos (por ejemplo: conseguir y sembrar plantas ornamentales o frutales), reportes de la condición o estado del tiempo (periódicos, televisión, internet), análisis de noticias, programas de televisión, anuncios publicitarios, visitas de campo guiadas (a parques nacionales, proyectos ambientales, viveros, uso de energía solar, etc.); dibujo (por ejemplo: croquis de la comunidad), simulacros (ante eventos sísmicos y volcánicos), proponer/ participar en campañas escolares (por ejemplo, de limpieza) y en la comunidad, mapa conceptual, ensayo, debate, foro, preparación y aplicación de encuestas y elaboración de carteles.

El docente en el plan de estudios de Ciencias (I y II Ciclos): su rol y desafíos

El MEP, en sus disposiciones pedagógicas, estableció que: "El docente no es un implementador del currículo, el docente es el principal tomador de decisiones curriculares en el aula" (MEP, 2020, p. 2; el resaltado es del original). De acuerdo con esta nueva visión filosófica y pedagógica, se coloca al educador con el nuevo rol de mediador/guía de los procesos 
Tabla 4

Nivel cuarto año: categorías de análisis y conceptos presentes en el programa de estudio de Ciencias para el II Ciclo por año escolar, eje temático y criterio de evaluación

\begin{tabular}{|c|c|c|c|c|c|c|c|c|}
\hline \multirow{2}{*}{ 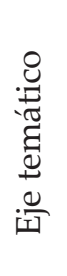 } & \multirow{2}{*}{$\begin{array}{c}\text { Código del } \\
\text { nivel, eje, sec- } \\
\text { ción y criterio } \\
\text { de evaluación }\end{array}$} & \multirow[b]{2}{*}{ Descripción del criterio de evaluación } & \multicolumn{4}{|c|}{$\begin{array}{c}\text { Conceptos y categorías } \\
\text { de análisis }\end{array}$} & \multicolumn{2}{|c|}{ Secciones } \\
\hline & & & 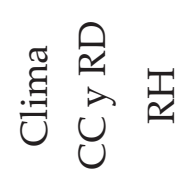 & $\frac{2}{0}$ & 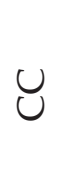 & $\begin{array}{l}u \\
u \\
0 \\
\frac{1}{0}>\end{array}$ & 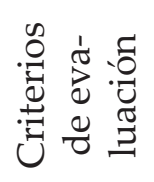 & 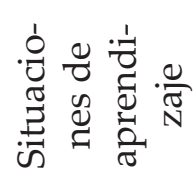 \\
\hline
\end{tabular}

I. $^{\mathbf{a}} \quad$ N4. ${ }^{\mathrm{o}} \mathrm{I}-5.1$, 1. Describir conceptos básicos relacio-

N4. ${ }^{\circ}$-5.2 nados con la biodiversidad, para un

N4..$^{\circ}$ 1-5.3 mejor entendimiento del entorno natural. 2. Explicar los aspectos que determinan la biodiversidad de Costa Rica y su importancia a nivel mundial. 3 . Tomar conciencia de los factores que amenazan la biodiversidad en la comunidad y su impacto para el país.

I. ${ }^{\text {b }} \quad$ N4..$^{\mathrm{o}}$ II-1.1 1 . Reconocer algunas clases de energía N4. ${ }^{\circ}$ II-1.2 que se manifiestan en el entorno natural N4. ${ }^{\circ}$ II-1.3 y su aplicación en la vida diaria. 2. Distinguir manifestaciones de la energía potencial y cinética, en situaciones cotidianas. 3 . $X \times X \quad X$ Apreciar la utilidad de la transformación de la energía potencial en energía cinética y viceversa, en situaciones cotidianas.

N4.. II-3.1 1. Reconocer las formas de transmisión N4..$^{\circ}$ II-3.2 del calor y la temperatura en situacioN4.. II-3.3 nes cotidianas. 2. Comprender la relación entre masa, calor y temperatura en situaciones cotidianas. 3. Valorar las medidas preventivas ante situaciones que involucren el uso del calor y la información que brindan las mediciones de la temperatura para evitar accidentes.

N4. ${ }^{\circ}$ II-4.1 1. Reconocer algunos de los fenómenos N4..$^{\circ}$ II-4.2 en los que interviene la luz y sus aplicaN4. ${ }^{\circ}$ II-4.3 ciones en la vida diaria. 2. Describir los fenómenos de reflexión y refracción de la luz en situaciones cotidianas, por medio de materiales pulidos, transparentes, $x \quad x \quad x \quad x \quad x$ translúcidos u opacos. 3. Valorar las medidas preventivas ante situaciones, en las cuales, la luz puede afectar al ser humano.

Continúa... 
III N4..$^{\mathrm{o}}$ III-1.1 1. Reconocer la estructura externa e inN4. ${ }^{\circ}$ III-1.2 terna del planeta Tierra y las actividades N4. ${ }^{\circ}$ III-1.3 que realiza la especie humana. 2. Determinar las influencias recíprocas entre el clima y las actividades que realiza la especie humana. 3 . Tomar conciencia de las causas y efectos de la contaminación atmosférica y del agua, para la implementación de medidas preventivas que salvaguarden el bienestar del planeta.

N4..$^{\circ}$ III-2.1 1. Reconocer los movimientos del pla- $X$

N4. ${ }^{\circ}$ III-2.2 neta Tierra y la Luna, como parte del N4. ${ }^{\circ}$ III-2.3 entendimiento de su vinculación con el Universo. 2. Explicar los eclipses de Luna y de Sol a partir de la representación de los movimientos que realiza el planeta Tierra y la Luna. 3. Apreciar la influencia de los movimientos del planeta Tierra y de la Luna en las actividades que realiza la especie humana y otros seres vivos.

Nota. Arauz, Charpentier y Moreira, 2020, con base en el análisis del Plan de Estudios de Ciencias de II Ciclo (Costa Rica, 2016).

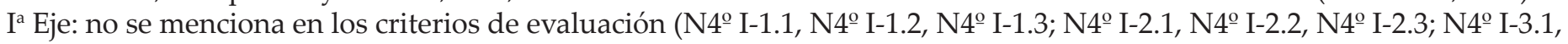
$\mathrm{N} 4^{\mathrm{o}} \mathrm{I}-3.2, \mathrm{~N} 4^{\mathrm{o}} \mathrm{I}-3.3$; N $4^{\mathrm{o}} \mathrm{I}-4.1$ y N $4^{\circ} \mathrm{I}-4.2, \mathrm{~N} 4^{\circ} \mathrm{O}$ I-4.3).

$\mathrm{II}^{\mathrm{b}}$ Eje: no se menciona en los criterios de evaluación (N4º II-2.1, N $4^{\circ} \mathrm{II}-2.2$ y N $\left.4^{\circ} \mathrm{II}-2.3\right)$.

\section{Tabla 5}

Nivel quinto año: categorías de análisis y conceptos presentes en el programa de estudio de Ciencias para el II Ciclo por año escolar, eje temático y criterio de evaluación

\begin{tabular}{|c|c|c|c|c|c|c|c|c|c|}
\hline \multirow{2}{*}{ 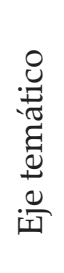 } & \multirow{2}{*}{$\begin{array}{l}\text { Código del } \\
\text { nivel, eje, sec- } \\
\text { ción y criterio } \\
\text { de evaluación }\end{array}$} & \multirow[b]{2}{*}{ Descripción del criterio de evaluación } & \multicolumn{5}{|c|}{$\begin{array}{c}\text { Conceptos y categorías } \\
\text { de análisis }\end{array}$} & \multicolumn{2}{|c|}{ Secciones } \\
\hline & & & 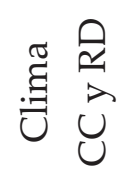 & $\underset{\mathbb{I}}{\mathbb{Z}}$ & $\frac{2}{0}$ & 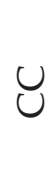 & $\begin{array}{l}u \\
u \\
0 \\
\frac{2}{u} \\
>\end{array}$ & 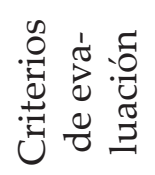 & 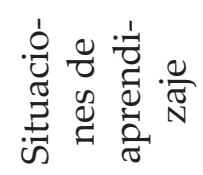 \\
\hline
\end{tabular}

I. ${ }^{\mathrm{a}} \quad$ N5. $.^{\mathrm{o}} \mathrm{I}-4.1$ 1. Reconocer, como parte del estudio de

N5. ${ }^{\circ}$ I-4.2 la biodiversidad, las características físi-

N5. ㄴ I-4.3 cas propias de algunos organismos que permiten clasificarlos de diferentes maneras. 2. Describir algunas relaciones de interdependencia entre los seres vivos y su importancia en el equilibrio ecológico. 3. Valorar las acciones personales y comunitarias dirigidas al uso racional de la flora y la fauna de la región. 
I. ${ }^{a} \quad$ N5..$^{\mathrm{O}}$ I-5.1 1 1. Reconocer la clasificación de los seres vivos toman-

N5. ${ }^{\circ}$ I-5.2 do en cuenta la forma de obtención de alimento, como

N5. ${ }^{-1-5.3}$ parte de la biodiversidad. 2. Describir el proceso de fotosíntesis que realizan las plantas como componentes esenciales del entorno natural. 3. Tomar conciencia sobre la importancia del proceso de la fotosíntesis para el mantenimiento de la vida en el planeta Tierra.

II $^{\text {B }} \quad$ N5. $\mathrm{o}$ II-1.1 1 . Identificar las aplicaciones de las diferentes N5.. II-1.2 clases de energía en las actividades cotidianas N5.. II-1.3 que se realizan en la comunidad. 2. Describir algunas transformaciones de la energía, utilizando diferentes materiales presentes en el entorno. 3. Apreciar el uso eficiente de las transformaciones de la energía, que posibilitan la realización de diferentes actividades en la vida cotidiana.

N5. II-3.3 3. Valorar las medidas de prevención de accidentes, relacionados con el uso de la energía eléctrica en la vida diaria.

N5.o II-4.1 1. Explicar las transformaciones de energía, que N5.. II-4.2 ocurren en la generación de electricidad, desde las N5. II-4.3 plantas hidroeléctricas hasta su uso en el hogar.

2. Describir los principales avances científicos y tecnológicos para la generación de energía eléctrica en Costa Rica. 3. Valorar las acciones dirigidas al uso racional de la energía eléctrica en la vida diaria y su relación con la protección del ambiente y el ahorro económico a nivel local y nacional.

III $\quad$ N5. ${ }^{\mathrm{o}}$ III-1.1 1 . Analizar los beneficios que puede obtener la esN5. III-1.2 pecie humana a partir de los efectos producidos por N5.. III-1.3 los agentes internos que modifican el relieve terrestre. 2. Describir los efectos recíprocos entre los agentes externos que modifican el relieve terrestre y las actividades que realiza la especie humana. 3. Tomar conciencia de la necesidad de implementar acciones y medidas preventivas ante los eventos sísmicos y volcánicos que ocurren en el país.

Nota. Arauz, Charpentier y Moreira, 2020, con base en el análisis del Plan de Estudios de Ciencias de II Ciclo (MEP, 2016).

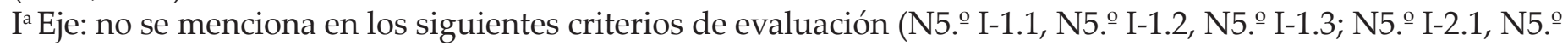

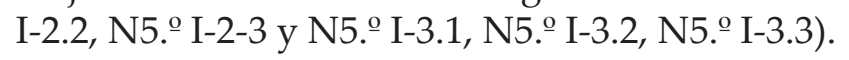

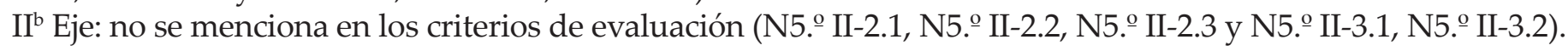

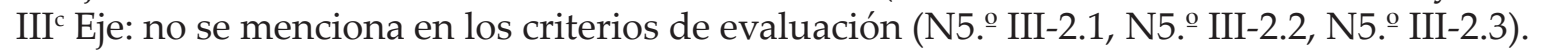


Tabla 6

Nivel sexto año: categorías de análisis y conceptos presentes en el programa de estudio de Ciencias para el II Ciclo por año escolar, eje temático y criterio de evaluación

\begin{tabular}{|c|c|c|c|c|c|c|c|c|}
\hline \multirow{2}{*}{ 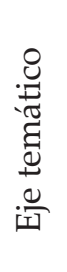 } & \multirow{2}{*}{$\begin{array}{c}\text { Código del } \\
\text { nivel, eje, sec- } \\
\text { ción y criterio } \\
\text { de evaluación }\end{array}$} & \multirow[b]{2}{*}{ Descripción del criterio de evaluación } & \multicolumn{4}{|c|}{$\begin{array}{c}\text { Conceptos y categorías } \\
\text { de análisis }\end{array}$} & \multicolumn{2}{|c|}{ Secciones } \\
\hline & & & 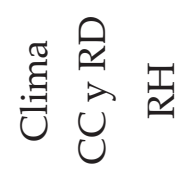 & $\frac{\theta}{0}$ & $\cup$ & $\begin{array}{l}u \\
u \\
\frac{1}{0} \\
0\end{array}$ & 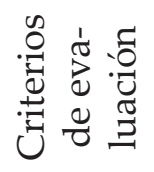 & 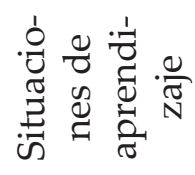 \\
\hline
\end{tabular}

I. ${ }^{\mathrm{a}} \quad \mathrm{N} 6 .^{\mathrm{o}} \mathrm{I}-4.1 \quad$ 1. Describir las interrelaciones entre los N6.. I-4.2 componentes de los ecosistemas, como N6.. I-4.3 parte del cuidado de la biodiversidad. 2. Distinguir los niveles de organización de los seres vivos, apreciando las relaciones que establecen en diferentes ecosistemas. 3. Valorar la diversidad de ecosistemas, paisajes y riqueza biológica de nuestro país, para su conservación y aprovechamiento sostenible.

N6.. I-5.1 1. Identificar los eventos naturales y las N6.. I-5.2 acciones humanas que alteran el equiN6. ${ }^{\circ}$ 1-5.3 librio ecológico. 2. Describir los efectos y consecuencias de las acciones provocadas por los eventos naturales y actividades humanas en la vida diaria. 3. Valorar las prácticas personales y comunitarias que contrarrestan los efectos negativos de los eventos naturales y actividades humanas para el fortalecimiento del desarrollo sostenible de Costa Rica.

II. ${ }^{\text {b N6. }}$ II-1.1 1. Describir la metodología utilizada N6.. II-1.2 en los avances científicos y tecnológiN6.. II-1.3 cos para el aprovechamiento de algunas clases de energía y su importancia para el desarrollo económico del país. 2. Distinguir las aplicaciones de la energía eólica en situaciones cotidianas y su impacto en el ambiente. 3. Tomar conciencia de los efectos causados por el uso de fuentes de energías contaminantes y no contaminantes en el ambiente. 


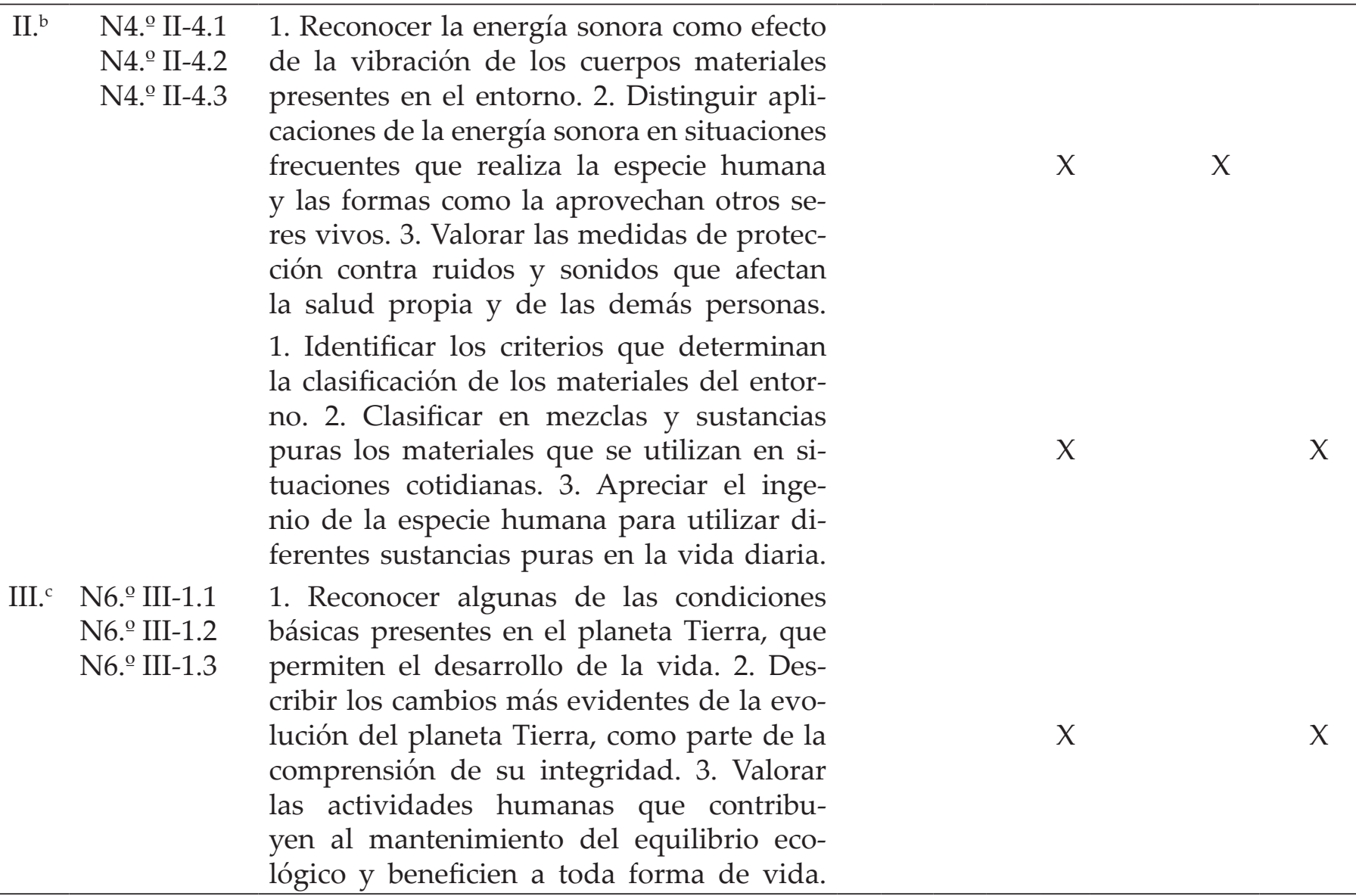

Nota. Arauz, Charpentier y Moreira, 2020, con base en el análisis del Plan de Estudios de Ciencias de II Ciclo (MEP, 2016).

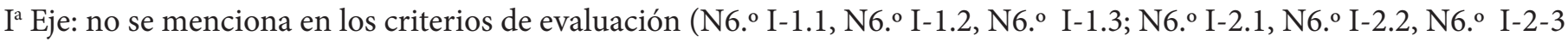
y N6. ${ }^{\circ}$ I-3.1, N6. ${ }^{\circ}$ I-3.2, N6. ${ }^{\circ}$ I-3.3).

$\mathrm{II}^{\mathrm{b}}$ Eje: no se menciona en los criterios de evaluación (N6. ${ }^{\circ}$ II-3.1, N6. ${ }^{\circ}$ II-3.2, N6. ${ }^{\circ}$ II-3.3).

III ${ }^{\mathrm{c}}$ Eje: no se menciona en los criterios de evaluación (N6. ${ }^{\circ}$ III-2.1, N6. ${ }^{\circ}$ III-2.2, N6. ${ }^{\circ}$ III-2.3).

educativos, además, se le otorga un papel de decisor, y se le asignan distintas obligaciones y competencias dentro de su perfil profesional. Dentro de los planes, se establecen quince habilidades para el perfil del educador; para ilustrarlo, la competencia catorce dice: "Planifica estrategias que permitan al estudiando colaborar en la resolución de problemas ambientales de la comunidad, comprendiendo su importancia para el bienestar de la región, del país y del planeta" (MEP, 2016, p. 33). Por lo tanto, si el educador no cuenta con esta competencia, no será capaz de desarrollar esta habilidad con sus estudiantes e incumpliría con las exigencias del MEP o, más importante aún, con el proceso trasformador que debe tener la educación en la vida de los estudiantes.

Es fundamental que el docente desarrolle las situaciones de aprendizaje considerando los conocimientos previos del alumno, el conocimiento del entorno natural, social y 
ambiental, y que comprenda las realidades circunscritas a diferentes escalas (local, nacional, planetaria) para el desarrollo de un aprendizaje contextualizado. Asimismo, la flexibilidad del currículum permite que los educadores puedan comparar la información derivada de distintas fuentes sobre los saberes presentes en los programas, así como ampliar y adaptar los tres ejes temáticos definidos oficialmente. Para esto, debe tener en cuenta variables como los horarios y el tipo de modalidad de la escuela, la disposición de recursos locales y las realidades socioculturales del sitio donde ejerce su trabajo (MEP, 2016).

Sin embargo, esta flexibilidad en el plan podría convertirse en una debilidad si el personal docente no se capacita o actualiza regularmente sobre los temas discutidos en este artículo. Por lo tanto, es urgente y necesario que, desde el $\mathrm{MEP}$, se continúen los esfuerzos de apoyo y acompañamiento pedagógico desde dos líneas. Primero, mediante capacitaciones curriculares para que los educadores aprendan teoría, fundamentos filosóficos y metodológicos sobre la nueva forma de elaborar el planeamiento didáctico en el marco de la mediación pedagógica, según el enfoque por competencias. Segundo, es necesario efectuar capacitaciones especializadas ${ }^{4}$, organizadas y articuladas con diferentes instancias y oficinas a lo interno del MEP. Tomando en cuenta las características de vulnerabilidad y riesgo de las diferentes regiones educativas; en este proceso podrían participar las universidades, el Consejo Nacional de Rectores (Conare), la Comisión Nacional de Emergencias (CNE), el Instituto Meteorológico Nacional, el Centro Nacional de Alta Tecnología (CeNAT), el Ministerio de Ciencia y Tecnología, la Dirección de CC, la Unesco, entre otros organismos públicos y privados, nacionales e internacionales.
Con ello, se estaría trabajando en atender las carencias existentes en la formación inicial de estos profesionales en temas tan álgidos para el desarrollo del país como la sostenibilidad. El éxito en la nueva reforma curricular pasa primero por atender estos desafíos y, en este sentido, se comparte el criterio de Portillo-Torres (2017), quien expone que al MEP todavía le falta atender algunos aspectos como "el manejo de tiempo, cantidad de estudiantes por docente, el tipo de evaluación, organización de los servicios de apoyo, entre otros" (p. 11).

La importancia de los conceptos analizados para el desarrollo de competencias ambientales y su armonización con los objetivos de desarrollo sostenible (ODS)

Los conceptos relacionados con CC y con GRD se corresponden con elementos importantes para el desarrollo de las competencias ambientales. Muchas de las nociones (elementos del clima, ciclo del agua, variabilidad climática y CC, amenazas naturales y antropogénicas, así como calentamiento global, entre otros) servirán como elementos clave en la construcción de saberes para la vida y la apropiación de las competencias en función de su nivel de desempeño (identificación, análisis, evaluación de soluciones latentes, y la propuesta y justificación de acciones

${ }^{4}$ Ejemplo de estos son: 1. "Curso virtual de gestión del riesgo de desastres para educadores", promovido por el Departamento de Control Interno y Gestión del Riesgo del MEP y la CNE con el apoyo del Centro de Coordinación para la Prevención de Desastres Naturales en América Central y República Dominicana (Cepredenac) y 2. "Curso prácticas pedagógicas en prevención de riesgos naturales", impartido por el Observatorio Vulcanológico y Sismológico de Costa Rica de la Universidad Nacional (Ovsicori-UNA). 
para resolver los problemas ambientales). La trascendencia de los conceptos analizados para el desarrollo de competencias ambientales por parte del estudiantado, y su correspondencia con los 17 ODS propuestos en la Agenda 2030 para el Desarrollo Sostenible de las Naciones Unidas, radica en que tanto la adaptación al CC (ACC) como la GRD son procesos de gestión complementarios de una misma agenda, cuyo fin es la reducción de la vulnerabilidad y la sostenibilidad del planeta.

Por lo tanto, se debe trabajar en los diferentes factores subyacentes del riesgo de desastre como la educación, la desigualdad, la pobreza, el CC y la variabilidad climática, entre otros impulsores representados en los ODS. En este contexto, entran en juego particularmente el ODS 4 "Educación de calidad", el ODS 6 "Agua limpia y saneamiento" y el ODS 13 "Acción climática" (PNUD, 2020), sin menospreciar el análisis integral de todos los demás. Otro punto es que estas metas son congruentes con el objetivo del Marco de Sendai para la Reducción del Riesgo de Desastres 2015-2030, tendiente a promover la seguridad de las escuelas y demás instalaciones para tener ambientes protectores. Para el efecto y eficacia pedagógica de los procesos de aprendizaje relacionados con la GRD y vinculados con el CC, estos se deben enfocar bajo la tríada dimensional del aprendizaje cognitivo, socioemocional y conductual contempladas tanto en las competencias generales del modelo propuesto por la NAAEE como en los ODS. El MEP debe fortalecer este aspecto para lograr la ejecución real de los planteamientos de los programas.

\section{Conclusiones}

En los perfiles de salida del estudiante de I y II ciclos en la asignatura de Ciencias, relacionados con el modelo de desarrollo sostenible, no se menciona ningún indicador definido que aborde concretamente el problema de cambio climático. Caso contrario ocurre con el tema de la GIRD, pues en el perfil de salida del estudiante de I Ciclo se encuentran dos indicadores que se corresponden con dicho término, aunque no de manera literal. El primero hace referencia a la comprensión, por parte de educando, de las medidas de prevención y protección en situaciones de emergencia originadas por la acción humana o por eventos naturales. El segundo se refiere al reconocimiento de diferentes tipos de riesgos socioambientales que afectan a todos los seres vivos del planeta.

El mayor énfasis temático con respecto a los conceptos sobre condiciones y estado del tiempo, información meteorológica en relación con fenómenos o eventos naturales y la toma de medidas de prevención en situaciones extremas está en el I Ciclo a lo largo de seis de las once secciones que componen los planes de estudio. En contraposición, solo en dos de las secciones del II Ciclo se menciona literalmente la palabra "clima" y, en ambas se vincula con medidas de prevención ante eventos naturales y provocados por la acción humana.

Los programas de estudio de Ciencias para I y II ciclos, según la óptica curricular, están conceptualizados desde la visión de un ciclo continuo de desarrollo, ejecución y evaluación. Esto permite fomentar habilidades básicas como el pensamiento crítico, sistémico y creativo, la comprensión de sistemas complejos, valores y actitudes sociales para la vida, el trabajo y el crecimiento personal de los estudiantes. Estas características presentes en los planes de estudio lo hacen un currículum de "buena calidad" (Stabbak, 2016, p. 11). No obstante, la realidad de la práctica docente dificulta que este salto cualitativo dado por el Estado costarricense se materialice en la formación de competencias ambientales en la población estudiantil. Por ello, es necesario trabajar en la elaboración de una propuesta educativa que coadyuve al proceso 
pedagógico del docente y de aprendizaje del estudiante para formar en tales competencias.

Sobre el análisis del contenido temático en los programas de estudio, se concluye que el concepto "riesgos socioambientales" es congruente con la actual concepción teórica de la GIRD, empleada por el corpus científico a nivel nacional y mundial. Esta enfatiza en el nuevo paradigma cultural relacionado con la gestión del riesgo de desastre, contrario al de la atención de la emergencia que ha predominado en las sociedades tanto en la teoría como en la práctica. Bajo esta visión curricular, los programas de estudio, desde el enfoque sistémico de la GIRD para el sector educativo y desde este, a nivel teórico contemplan la etapa de la reducción del riesgo, la cual es la principal acción de este complejo proceso. Además, en cuanto a la reducción en el riesgo de desastres y la educación resiliente, se cumple con una de las tres condiciones básicas: la integración en el currículum educativo de estas importantes temáticas. Pese a lo anterior, se deben sumar las demás etapas: la de protección de la infraestructura escolar (medidas estructurales) y la de preparativos y elaboración de planes de seguridad del centro educativo (medidas no estructurales de prevención y mitigación) para tener a la población estudiantil, administrativa y docente completamente segura.

Para el II Ciclo escolar, en la sección denominada "situaciones de aprendizaje", se le presentan al docente los vocablos: clima, efectos del calentamiento global, cambios en el clima, estado del tiempo, CC, gases de efecto invernadero, destrucción de la capa de ozono, prevención de situaciones vulnerables, eventos o fenómenos naturales (inundaciones, sequías, corrientes de resaca), amenazas naturales y humanas, fenómenos humanos, agua, atmósfera, recursos hídricos e hidrosfera. Se destaca que los programas de estudio están elaborados bajo el enfoque de indagación y se presentan en el documento preguntas de apoyo al docente en el proceso de mediación pedagógica, esto para fomentar el desarrollo de la competencia mediante la propuesta de preguntas dinamizadoras que impulsen el pensamiento crítico y reflexivo de las temáticas bajo estudio. También se propone que los estudiantes trabajen en grupos para dar respuestas a varias preguntas y luego, en plenaria, presenten sus ideas; aparte, deben tomar notas sobre el objeto de estudio, entre otras técnicas de aprendizaje. Por ejemplo, en el plan se plantean preguntas que establecen la relación entre el tema de la energía con el calentamiento global.

La presencia de ciertos contenidos temáticos está en consonancia con el modelo teórico de la alfabetización ambiental de la NAAEE (Hollweg et al., 2011), que promueve una clasificación de los contextos ambientales y de diferentes escalas geográficas (local, nacional y global) en la esfera educativa, así como la recomendación de incluir el estudio de los riesgos de desastres y los eventos climáticos extremos en los diferentes años escolares de la educación formal. De esta manera, por ejemplo, a nivel local recomienda tener en cuenta la temática de planificación y toma de decisiones sobre la construcción en áreas vulnerables por inundación, por efecto de las mareas y por vientos huracanados; a escala nacional, promueve el estudio de cambios vertiginosos, como los sismos, y los procesos geomorfológicos más lentos, como la erosión costera, al igual que los riesgos y beneficios asociados a estas dinámicas terrestres.

En resumen, es posible afirmar que Costa Rica está cumpliendo a nivel prescriptivo - mediante la política educativa y curricular- con la adopción de las estrategias definidas por el IPCC de la adaptación al CC, así como con la GIRD determinada en el Marco de Sendai, ambas se enfocan en reducir la vulnerabilidad causada por 
el CC para favorecer a la niñez. Sumado a ello, el país es coherente con el esfuerzo internacional por establecer vínculos entre las agendas para la reducción de riesgos de desastres (RRD) y del $\mathrm{CC}$, así como del consenso para que una de las principales acciones por realizar sea introducir el tema de RRD y CC en la educación formal y no formal, lo cual incluye todas las actividades relacionadas con estos asuntos críticos para la sociedad de hoy.

Sin embargo, entendiendo que se trata de un proceso y esfuerzo constante y paulatino, el país debe seguir trabajando en la adopción de medidas necesarias para mejorar la educación, la sensibilización, la participación de la sociedad y el acceso público a la información sobre el CC. Por ello, es necesario evaluar en las aulas la puesta en marcha de los programas que incorporan el estudio del CC y la ocurrencia de eventos hidrometeorológicos extremos, con el fin de reducir la vulnerabilidad y aumentar la resiliencia que mejoraría la calidad de vida de la población infantil costarricense.

\section{Agradecimientos}

Se extiende un profundo agradecimiento al programa CeNAT-Conare por el financiamiento otorgado, mediante la Beca de Investigación 2019, a la primera autora del artículo. También, se reconoce la valiosa colaboración de la Ing. Elizabeth Solís Quirós, asesora nacional de Ciencias del Ministerio de Educación Pública en la revisión del documento.

\section{Referencias}

Asamblea Legislativa de la República de Costa Rica (ALRCR). (2006). Ley N. 8488 Ley Nacional de Emergencias y Prevención del Riesgo. Diario Oficial La Gaceta 8.

Alfaro-Rodríguez, M. (2015). ¿Por qué un currículo por habilidades en la reforma curricular de Costa Rica? En Transformación curricular: fundamentos conceptuales en el marco de la visión "educar para una nueva ciudadanía" (pp. 111-116). Costa Rica: MEP.

Chen-Quesada, E., Hernández- Chaves, C., Muñoz- Valenciano, E., y Segura- Bonilla, O. (2018). Desafíos de la política educativa para el desarrollo sostenible en Costa Rica. Revista Latinoamericana de Educación Comparada, 9(14), 105-122.

Delors, J. (1996). La educación encierra un tesoro. Delors, Francia: Unesco.

Espinoza-Villalobos, D., Fallas-Moya, L., López-Castillo, C., y Madrigal-Rojas, V. (2018). Guía pedagógica para niños y niñas desde el nacimiento hasta los 4 años. San José, Costa Rica: Dirección de Desarrollo Curricular MEP.

Fundación Omar Dengo. (2014). Competencias del siglo XXI: guía práctica para promover su aprendizaje y evaluación. Capítulo Latinoamericano del Proyecto Atc210s. Proyecto Atc21s. San José, Costa Rica: Fundación Omar Dengo.

Girot, P., y Perea, A. K. (2019). Gestión de riesgo de desastres y adaptación al cambio climático en Centroamérica. En Banco Mundial (Eds.), Informe Hacia Una Centroamérica Más Resiliente. Pilares para La Acción, (pp. 1-205). Washington, DC, EE. UU.: Banco Mundial.

González-Gaudiano, E., y Meira-Cartea, P. (2020). Educación para el cambio climático: 
¿educar sobre el clima o para el cambio? Perfiles Educativos, 42(168), 157-174.

Hernández-Sampieri, R.; Fernández-Collado, C.; Baptista-Lucio, P. (2014). Metodología de la investigación (6. ㄹ ed.). México: McGraw-Hill.

Hollweg, K. S., Taylor, J. R., Bybee, R. W., Marcinkowski, T. J., McBeth, W. C., y Zoido, P. (2011). Developing a framework for assessing environmental literacy. Washington, DC, EE. UU.: North American Association for Environmental Education.

IPCC. (2013). Glosario [Planton, S. (ed.)]. En: Cambio Climático 2013. Bases físicas. Contribución del Grupo de trabajo I al Quinto Informe de Evaluación del Grupo Intergubernamental de Expertos sobre el Cambio Climático [Stocker, T. F., D. Qin, G.-K. Plattner, M. Tignor, S. K. Allen, J. Boschung, A.; Nauels, Y.; Xia, V.; Bex y P. M. Midgley (eds.)]. Cambridge: Cambridge University Press.

IPCC. (2014). Cambio climático 2014: impactos, adaptación y vulnerabilidad. Resumen para responsables de políticas. GT II Contribución del Grupo de Trabajo al Quinto Informe de evaluación del IPCC. Ginebra, Suiza: OMM y PNUMA.

IPCC. (2019). Summary for policymakers. En H. O. Pörtner, D. C. Roberts, V. Masson-Delmotte, P. Zhai, M. Tignor, E. Poloczanska, K. Mintenbeck, M. Nicolai, A. Okem,

J. Petzold, B. Rama y N. Weyer (eds.), IPCC special report on the ocean and cryosphere in a changing climate. En prensa.

Ireland, L. (2013). Environmental education framework: pathways to environmental literacy in Alberta schools, supporting Alberta education's curriculum redesign. Canadá: Alberta Council for Environmental Education.

Marope, M., Griffin, P., y Gallagher, C. (2018). Future competences and the future of curriculum: a global reference for curricula transformation. Ginebra, Suiza: International Bureau of Education.

Marzano, R. J., y Kendall, J. S. (2007). The new taxonomy of educational objectives. (2. a ed.). Thousand Oaks, C.A.: Corwin Press A Sage Publications Company.

Ministerio de Educación Pública (MEP, 2009).

La planificación y la mediación pedagógica desde el enfoque de la educación científica: módulo 2. Costa Rica: MEP.

Ministerio de Educación Pública (MEP, 2014). Orientaciones estratégicas 2015-2018. Costa Rica: MEP.

Ministerio de Educación Pública (MEP, 2015a). Transformación curricular: fundamentos conceptuales en el marco de la visión "Educar para una nueva ciudadanía". Costa Rica: MEP, Viceministerio Académico.

Ministerio de Educación Pública (MEP, 2015b). Fundamentación pedagógica de la transformación curricular 2015: educar para una nueva ciudadanía. Costa Rica: MEP.

Ministerio de Educación Pública (MEP, 2016). Programas de estudio de ciencias primero y segundo ciclos de la educación general básica. Costa Rica: MEP.

Ministerio de Educación Pública (MEP, 2017). Política educativa: la persona: centro del proceso educativo y sujeto transformador de la sociedad. Costa Rica: MEP.

Ministerio de Educación Pública (MEP, 2020). Lineamientos generales para el inicio del curso lectivo 2020. "Año 2020: transformación curricular, una apuesta por la calidad educativa. Acuerdo CSE-03-01-2020". Circular DM0004-01-2020 del 30 de enero de 2020. Costa Rica: MEP.

Ministerio de Educación Pública y Consejo Superior de Educación del (CSE). (2016). Oficio 
CSE-SG-0516-2016. Acuerdo N.ํo 03-30-2016. Aprobar los programas de estudio de Ciencias de I y II Ciclo de la Educación General Básica. Costa Rica: MEP.

Ministerio de Planificación Nacional y Política Económica (Mideplan, 2014). Plan Nacional de Desarrollo 2015-2018 "Alberto Cañas Escalante". Costa Rica: Mideplan.

Ministerio de Planificación Nacional y Política Económica (Mideplan, 2015). Costa Rica: objetivos de desarrollo del milenio, III informe país 2015. Costa Rica: Mideplan.

Organización de las Naciones Unidas. (2015). Resolución aprobada por la Asamblea General el 25 de septiembre de 2015. 70/1. Transformar nuestro mundo: la Agenda 2030 para el Desarrollo Sostenible Documento A/70/L.1, 18 de septiembre de 2015. Nueva York, EE. UU.: ONU.

Organización para la Cooperación y el Desarrollo Económicos (OCDE). (2017). Educación en Costa Rica 2017: mejores políticas para una vida mejor. París, Francia: OCDE.

Organization for Economic Cooperation and Development (OECD). (2017). Education in Costa Rica. Basic education in Costa Rica: from access to learning for all. En Education in Costa Rica, Reviews of National Policies for Education (99-136). París, Francia: OCDE.

Programa de la Naciones Unidas para el desarrollo (PNUD, 2020). Objetivos de Desarrollo Sostenible. Programa de las Naciones Unidas para el Desarrollo. Recuperado de https://www.undp.org/content/undp/es/ home/sustainable-development-goals.html

Portillo-Torres, M. (2017). Educación por habilidades: Perspectivas y retos para el sistema educativo. Revista Educación. 41(2), 1-13.

Romero-Ariza, M. (2017). El aprendizaje por indagación: ¿existen suficientes evidencias sobre sus beneficios en la enseñanza de las ciencias? Revista Eureka sobre Enseñanza y Divulgación de las Ciencias, 14(2), 286-299.

Stabbak, P. (2016). Qué hace un currículo de calidad. IBE/2016/WP/CD/02. Oficina Internacional de Educación de la Unesco. Ginebra, Suiza: IBE.

Torres-Arias, R., Barrantes-Benavides, J., Leitón-Céspedes, O., Marchena-López, J., Mora-Guillén, I., y Quesada-Carvajal, R. (2015). Respuestas a las consultas más frecuentes en el proceso de evaluación de los aprendizajes. San José, Costa Rica: MEP.

Organización de las Naciones Unidas para la Educación, la Ciencia y la Cultura (Unesco, 2010). Herramienta analítica 9 de la lente de la EDS: la integración de la EDS en los planes de estudios. La lente de la EDS: una herramienta para examinar las políticas y la práctica. Instrumentos de Aprendizaje y Formación, N.o 2. París, Francia: Unesco.

Organización de las Naciones Unidas para la Educación, la Ciencia y la Cultura (Unesco, 2012). Educación para el desarrollo sostenible. Libro de consulta. Instrumentos de aprendizaje y formación N. ${ }^{\circ} 4-2012$. La Educación para el Desarrollo Sostenible en acción. París, Francia: Unesco.

Organización de las Naciones Unidas para la Educación, la Ciencia y la Cultura (Unesco, 10 al 12 de noviembre de 2014). Declaración de Aichi-Nagoya sobre la educación para el desarrollo sostenible. Recuperado de https://unesdoc.unesco. org/ark:/48223/pf0000231074_spa

Organización de las Naciones Unidas para la Educación, la Ciencia y la Cultura (Unesco, 2014). Roadmap for implementing the global action programme on education for sustainable development. París, Francia: Unesco.

Organización de las Naciones Unidas para la Educación, la Ciencia y la Cultura (Unesco, 2015). Not just hot air: putting climate change education into practice. París, Francia: Unesco. 
Organización de las Naciones Unidas para la Educación, la Ciencia y la Cultura (Unesco, 2017). Educación para los Objetivos de Desarrollo Sostenible. Objetivos de aprendizaje. París, Francia: Unesco.

Recibido: 24 de marzo de 2020

Aceptado: 16 de julio de 2020 